\title{
IDEALS OF OPERATORS STRICTLY SINGULAR ON SUBSPACES
}

\author{
MICHAEL J. MEYER
}

(Communicated by Palle E. T. Jorgensen)

\begin{abstract}
For each set $\Omega$ of cardinality at most $2^{\omega}$, we give an easy construction of Banach spaces $X$, such that the algebra $\mathscr{B}(X)$ of all bounded linear operators on $X$ contains a lattice of closed ideals, which is order isomorphic with respect to inclusion to the full power set of $\Omega$.
\end{abstract}

\section{INTRODUCTION AND RESULT}

Let $X$ be a Banach space and $\mathscr{B}(X)$ the Banach algebra of bounded linear operators on $X$. The ideal structure of $B(X)$ depends very much on the Banach space $X$. For example, if $X=c_{0}$ or $X=l_{p}, 1 \leq p<\infty$, then $B(X)$ contains only one nontrivial closed two-sided ideal, the ideal of compact operators.

On the other hand Porta constructed in [1] a Banach space $X$ and, for each nonempty finite subset $A \subseteq \mathbb{N}$, a closed two-sided ideal $H(A) \subseteq \mathscr{B}(X)$ such that the map $H: A \rightarrow H(A)$ is an order isomorphism with respect to set inclusion of the family of finite subsets $A \subseteq \mathbb{N}$ with the family of closed ideals $H(A) \subseteq \mathscr{B}(X)$.

In this note we give an extremely easy construction of a lattice of closed operator ideals on a suitable Banach space $X$, which is order isomorphic to the full power set of certain infinite sets $\Omega$.

Let us call two Banach spaces $E, F$ incomparable, if each bounded linear operator $T: E \rightarrow F$ is strictly singular [2, 2.c.1 and 2.c.2]. With this we can show:

Theorem 1. Suppose that the Banach space $X$ contains a family $\left(E_{\alpha}\right)_{\alpha \in \Omega}$ of pairwise incomparable complemented infinite-dimensional subspaces $E_{\alpha} \subseteq X$. Then for each subset $A \subseteq \Omega$ a closed two-sided ideal $I(A) \subseteq \mathscr{B}(X)$ can be defined such that the map $I: A \rightarrow I(A)$ is an order isomorphism with respect to inclusion of the power set of $\Omega$ with a family of closed ideals $I(A) \subseteq \mathscr{B}(X)$.

Proof. For each closed subspace $E \subseteq X$ let $S(E)$ denote the family of all operators $t \in B(X)$ such that the restriction $\left.t\right|_{E_{1}}: E_{1} \rightarrow X$ is strictly singular for each closed subspace $E_{1} \subseteq X$, which is isomorphic to $E$.

It is easily checked that $S(E)$ is a closed left ideal in $B(X)$. We claim that it is in fact a two-sided ideal. Since every element $a$ in the unital Banach algebra

Received by the editors March 16, 1993.

1991 Mathematics Subject Classification. Primary 47D50.

Key words and phrases. Operator ideals, strictly singular operators. 
$B(X)$ can be written as the sum of two invertibles $(a=\lambda 1+(a-\lambda 1)$, where $|\lambda|$ exceeds the spectral radius of $a)$, it will suffice to show that $S(E) g \subseteq S(E)$ for all invertibles $g \in \mathscr{B}(X)$.

Let now $t \in S(E)$ and $g \in \operatorname{inv}(B(X))$. If $E_{1} \subseteq X$ is any closed subspace isomorphic to $E$, then so is the subspace $g\left(E_{1}\right)$. Consequently the restriction $\left.t\right|_{g\left(E_{1}\right)}$ is strictly singular. But then the restriction $\left.t g\right|_{E_{1}}$ is strictly singular. This shows that $\operatorname{tg} \in S(E)$.

For each index $\alpha \in \Omega$ choose a projection $p_{\alpha} \in \mathscr{B}(X)$ with $p_{\alpha}(X)=E_{\alpha}$ and define

$$
I(A)=\bigcap_{\alpha \in \Omega \backslash A} S\left(E_{\alpha}\right), \quad \text { for each subset } A \subseteq \Omega,
$$

where $I(A)=\mathscr{B}(X)$, if $A=\Omega . I(A)$ is an intersection of closed two-sided ideals in $B(X)$ and hence itself such an ideal. Clearly $A \subseteq B \subseteq \Omega$ implies $I(A) \subseteq I(B)$. We claim now that

$$
p_{\alpha} \in I(A) \Leftrightarrow \alpha \in A, \quad \text { for all subsets } A \subseteq \Omega \text { and } \alpha \in \Omega .
$$

Note first that $p_{\alpha} \notin S\left(E_{\alpha}\right)$, since the restriction of $p_{\alpha}$ to the subspace $E_{\alpha}$ is not strictly singular. Thus $p_{\alpha} \notin I(A)$, if $\alpha \in \Omega \backslash A$. In other words, $p_{\alpha} \in I(A) \Rightarrow \alpha \in A$.

Conversely assume that $\alpha \in A$. We claim that $p_{\alpha} \in I(A)$ and must show that $p_{\alpha} \in S\left(E_{\beta}\right)$ for all $\beta \in \Omega \backslash A$. Indeed, we show that $p_{\alpha} \in S\left(E_{\beta}\right)$ for all $\beta \neq \alpha$.

Suppose that $\beta \in \Omega, \beta \neq \alpha$, and $F$ is any closed subspace of $X$ which is isomorphic to $E_{\beta}$. The projection $p_{\alpha}$ maps $F$ into $E_{\alpha}$. Since the spaces $E_{\beta}, E_{\alpha}$ and hence the spaces $F, E_{\alpha}$ are incomparable by assumption, the restriction of $p_{\alpha}$ to $F$ must be strictly singular. This shows that $p_{\alpha} \in E_{\beta}$ and proves (1). According to (1)

$$
A=\left\{\alpha \in \Omega: p_{\alpha} \in I(A)\right\} \quad \text { for each subset } A \subseteq \Omega .
$$

Thus $I(A) \subseteq I(B)$ implies $A \subseteq B$ for all subsets $A, B \subseteq \Omega$. This concludes the proof.

Remarks. The Banach spaces $E_{p}=l_{p}, 1 \leq p<\infty$, are well known to be pairwise incomparable [2, 2.c.1 and 2.c.2]. Suppose now that $\Omega$ is a set of cardinality at most $2^{\omega}$. We may assume that $\Omega$ is a subset of the interval [2, 3]. Then the Banach space

$X=\left\{x=\left(x_{p}\right)_{p \in \Omega}: x_{p} \in l_{p}\right.$, for all $p \in \Omega$, and $\left.\|x\|=\left(\sum_{p \in \Omega}\left\|x_{p}\right\|_{l_{p}}^{2}\right)^{1 / 2}<\infty\right\}$

satisfies the assumptions of Theorem 1 for the family of subspaces $\left(E_{p}\right)_{p \in \Omega}$. Theorem 1 now gives a large family of closed ideals in the Banach algebra $\mathscr{B}(X)$. It is now interesting to note that

Proposition 1. Let $X$ be a Banach space, and suppose that $I, J \subseteq \mathscr{B}(X)$ are distinct ideals which are Banach algebras in some norms. Then $I$ and $J$ are not isomorphic as complex algebras. 
Proof. According to [3, Theorem 2.5.19] any algebra isomorphism $\phi: I \rightarrow J$ is the restriction to $I$ of an inner automorphism of $\mathscr{B}(X)$. Consequently $\phi$ leaves $I$ invariant.

\section{ACKNOWLEDGMENTS}

The author expresses his gratitude to his former advisor Theodore Palmer for valuable discussions.

\section{REFERENCES}

1. H. Porta, Two sided ideals of operators, Bull. Amer. Math. Soc. 75 (1969), 599- 602.

2. J. Lindenstrauss and L. Tzafriri, Classical Banach spaces. I, Springer-Verlag, Heidelberg and New York, 1977.

3. C. E. Rickart, General theory of Banach algebras, van Nostrand, New York, 1960.

Department of Mathematics and Computer Science, Georgia State University, AtLANTA, GEORGIA 30303 